\title{
Antidotes edge closer to reversing effects of new blood thinners
}

The US approval late last year of the blood thinner Eliquis (apixaban) made it the third blood-thinner pill option to come online in the last three years, all of which have proven to be as or more effective as the old go-to warfarin, with lower rates of brain hemorrhaging and fewer blood tests required to get the dosing right. However, unlike warfarin, which has an antidote in the form of vitamin $\mathrm{K}$, no compounds exist that directly counteract the anticoagulation effects of these next-generation blood thinners-something desperately needed for the $1-3 \%$ of patients who suffer major bleeding complications, experience trauma injuries or require urgent surgery while on these pills.

New anticoagulation antidotes could be on the way. On 5 February, Portola Therapeutics, a South San Francisco-based company with an injectable antidote called PRT064445, announced that it had sealed a deal with Germany's Bayer and New Jersey's Janssen Pharmaceuticals to test PRT064445 in a phase 2 trial involving healthy volunteers given Xarelto (rivaroxaban). This pact comes on top of a similar partnership announced in November 2012 between Portola and the makers of Eliquis, Bristol-Myers Squibb and Pfizer, both based in New York.

Approved by the US Food and Drug Administration in 2011, Xarelto, like Eliquis, blocks a key enzyme in the blood-clotting cascade called factor Xa. PRT064445 works by acting as a decoy for drugs like Eliquis and Xarelto to bind to, preventing them from obstructing factor Xa in the bloodstream.

Reporting online in this journal on 3 March, Portola scientists showed in human plasma that PRT064445 reversed the anticoagulant effects of these drugs and another experimental factor Xa targeted agent known as betrixaban, as well as two indirect factor Xa inhibitors, including a form of heparin, an older injectable anticoagulant. The drug also restored normal clotting in rabbits and rats (Nat. Med. doi:10.1038/ $\mathrm{nm} .3102,2013)$. According to John Curnutte, head of research and development at Portola, based in South San Francisco, California, PRT064445 also proved safe and well tolerated in an as-yet-unpublished phase 1 study involving 32 healthy volunteers not on anticoagulant therapy.

"We want this drug to be available for those circumstances where the physicians, the surgeons and the emergency room staff feel it's really important to remove that blood

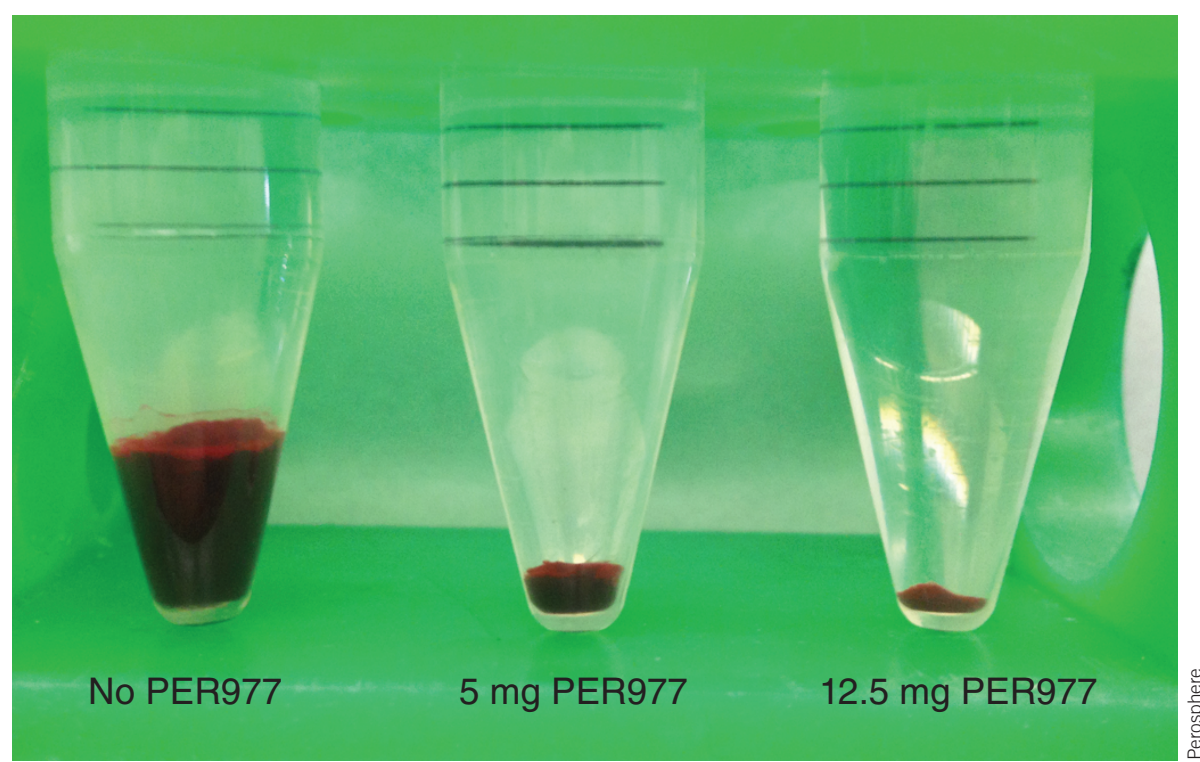

Staunch support: PER977 lessens blood loss in rats on Eliquis following a common bleeding assay.

thinner headwind that leads to this enhanced bleeding," Curnutte says, "and thereby enable them to manage the patients as if they were not on the anticoagulant."

\section{Coagulation countermeasures}

Other anticoagulation antidotes are nearing clinical testing, too. For example, Boehringer Ingelheim, the German company behind Pradaxa (dabigatran), a blood thinner approved since 2010 that inhibits factor IIa (also known as thrombin), is developing its own antidote in house. In December, at the American Society of Hematology meeting in Atlanta, company scientists presented preclinical data showing that treatment with an experimental antibody fragment against Pradaxa resulted in safe and rapid reversal of blood loss in rats. The drug is now in phase 1 testing.

Meanwhile, Perosphere, a startup headquartered in the suburbs of New York, has an antidote drug called PER977 that might reverse the activity of all the new oral anticoagulation drugs-both the factor Xa and factor IIa inhibitors-as well as certain forms of heparin. That broad action provides a major advantage, says Solomon Steiner, chief executive of the company. "When someone comes into an emergency room, they might not know exactly what they're on. If you have something that's going to work regardless, that gives a physician a lot more confidence in applying it."

Plus, as a synthetic small molecule,
PER977, unlike biologics such as PRT064445 in its current form, is stable at room temperatures. According to Sasha Bakhru, chief technology officer at Perosphere, that means "you can keep it in solution ready on an ambulance." Reporting at last year's American Heart Association Scientific Sessions meeting in Los Angeles, Bakhru, Steiner and their colleagues showed that PER977 reduced blood loss in rats treated with Eliquis, Xarelto, Pradaxa or another factor Xa inhibitor called Lixiana (edoxaban), which is approved in Japan, and produced no adverse effects in beagle dogs. The company plans to launch phase 1 human testing later this year.

Ideally one antidote drug that works for all the new blood thinners would be best because "shelf space is always an issue," says Edith Nutescu, director of the Antithrombosis Center at the University of Illinois at Chicago. But really, any reversing agent could be a huge help for emergency room doctors and perhaps "raise the comfort level" for prescribing physicians, she says.

According to William Dager, a pharmacist specialist at the University of CaliforniaDavis Medical Center's Anticoagulation Clinic, the vast majority of clinicians have been reluctant to switch to the new warfarin replacements. But, he says, "once we have better monitoring tests and the ability to reverse them when we have bleeding, I think there's going to be acceptance."

Elie Dolgin 